\title{
Accidental finding of a giant right coronary artery aneurysm associated with a fistula to the right atrium
}

\author{
Patrícia Vaz Silva, Joana Castro Marinho, António Pires \\ Pediatric Cardiology Department, Pediatric Hospital Carmona da Mota, CHUC EPE, Coimbra, Portugal
}

\begin{abstract}
Coronary artery fistulae are uncommon but may be haemodynamically significant, being an incidental finding in $0.1-0.2 \%$ of coronary angiograms. Even rarer is the association between fistulae and nonatherosclerotic coronary artery aneurysms. They most frequently originate in the right coronary artery, and the right cardiac chambers are the most common draining chambers. Most children are asymptomatic, whereas those older than 20 years may present with signs of congestive heart failure, infective endocarditis, myocardial ischaemia, or aneurysm rupture. Management is either surgical or via percutaneous means. We report the case of a 5-year-old child referred for assessment of an asymptomatic cardiac murmur. The echocardiographic evaluation showed an enlarged right atrium, a fenestrated atrial septal defect, and a giant right coronary artery aneurysm with a fistulous tract that appeared to drain directly into the right atrium. Computed angiocardiac tomography and cardiac catherisation confirmed the presence of a large right coronary fistula originating from the right coronary aneurysm draining into the right atrium. The patient underwent surgical ligation of the fistula and the post-operative course has been uneventful. He is currently on double antiaggregation therapy.
\end{abstract}

Keywords: Right coronary aneurysm; coronary fistula; fenestrated atrial septal defect

Received: 11 February 2013; Accepted: 29 March 2013; First published online: 22 May 2013

\section{Case report}

A 5-YEAR-OLD BOY WAS REFERRED TO OUR department for evaluation of a heart murmur. He had no relevant past medical history, was asymptomatic from a cardiac point of view, and was under no medication. On auscultation, a continuous murmur loudest at the left fourth intercostal space, with no radiation, was evident. On examination, no other relevant findings were found. His chest X-ray revealed an increased cardiothoracic ratio (65\%), with right-sided prominence heart and normal pulmonary vascular markings. The electrocardiogram at rest showed right-axis deviation (QRS complex axis of $+100^{\circ}$ ), associated with right atrium and right ventricle hypertrophy. Findings on two-dimensional

Correspondence to: P. Vaz Silva, Av. Afonso Romão, Santo António dos Olivais 3000-602 Coimbra, Portugal. Tel: +351 239488700; Fax: +351 239717216; E-mail: patriciavazsilva@gmail.com transthoracic echocardiogram included a moderately dilated right atrium and right ventricle, a giant aneurysm measuring $10 \mathrm{~mm}$ ( $Z$-score 19.6, Fig 1a) at the proximal portion of right coronary artery, normal origin and dimensions of the left coronary artery and a fistulous tract originating in the aneurysm and apparently draining into the right atrium next to the atrial septum (Fig 1b). On colour Doppler, a continuous turbulent flow into the right atrium was observed (Fig 1c). Biventricular function was preserved with normal regional motility, and the pulmonary artery pressures were normal. In order to clarify the coronary anatomy, a cardiac computed tomography angiogram (Fig 2) and cardiac catheterisation were performed, both confirming the above findings, as well as another aneurysm at the distal end of the fistula, measuring $15 \mathrm{~mm}$. The Qp/Qs was 2 .

The patient underwent surgical fistula ligation and closure of the fenestrated atrial septal defect. His post-operative period was uncomplicated and he 

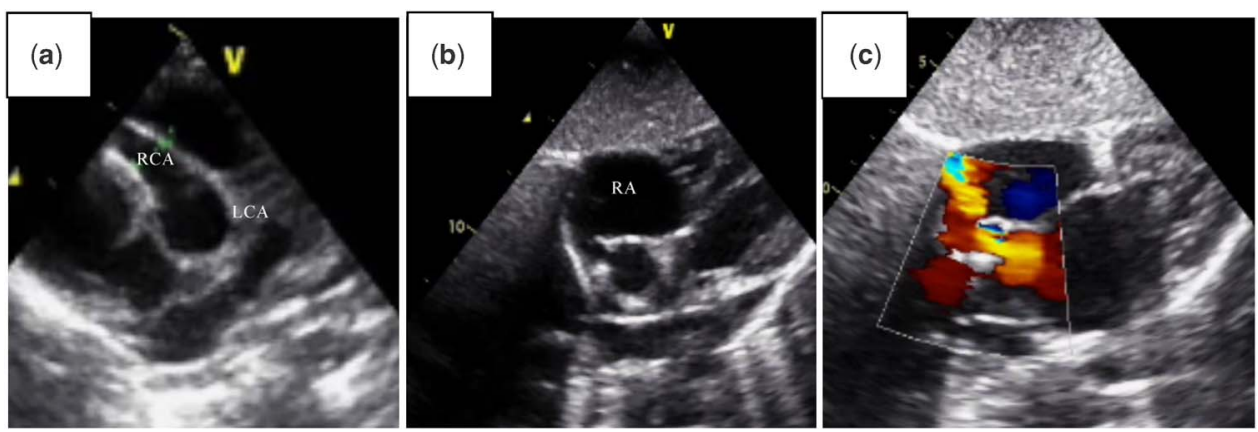

Figure 1.

Transthoracic echocardiogram showing an aneurysm of the proximal portion of the right coronary artery measuring about $10 \mathrm{~mm}(\mathrm{a})$. A 5-mm fistulous tract draining into the right atrium next to the atrial septum was identified (b), and related to a continuous turbulent flow (c). $L C A=$ left coronary artery; $R A=$ right atrium; $R C A=$ right coronary artery.
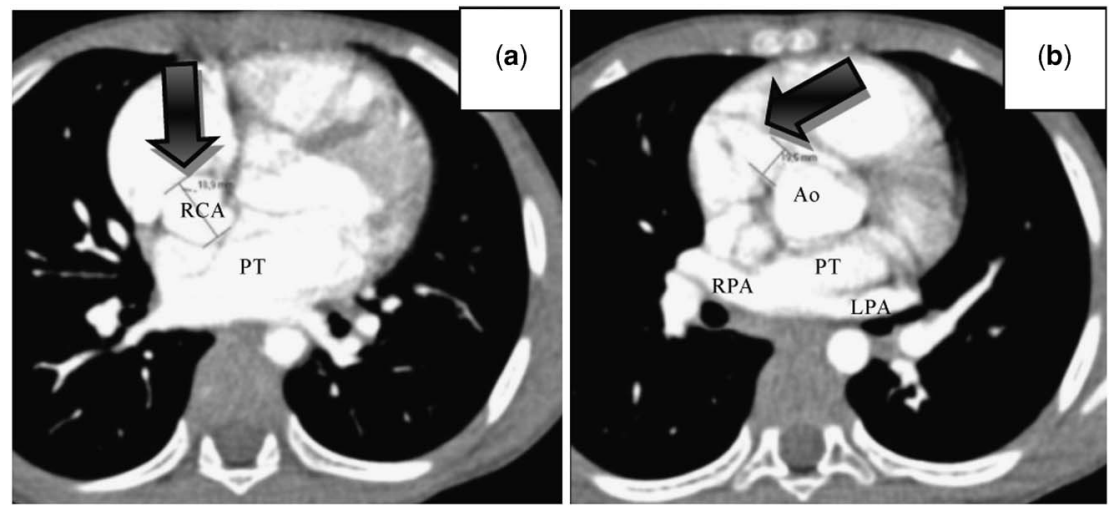

Figure 2.

Cardiac computed tomography angiogram showing a significantly dilated proximal right coronary artery (arrow, a), related to an anomalous communication compatible with a coronary fistula measuring $19 \mathrm{~mm}$ at its maximum diameter (arrow, b), that drains into the right atrium. $A_{0}=$ ao root; $L P A=$ left pulmonary artery; $P T=$ pulmonary trunk; $R C A=$ right coronary artery; $R P A=$ right pulmonary artery.

was discharged home 1 week after surgery on double antiaggregation therapy, with aspirin $(5 \mathrm{mg} / \mathrm{kg} /$ day $)$ and dipyridamole $(1 \mathrm{mg} / \mathrm{kg} /$ day $)$.

Currently, 6 months post surgery, he remains asymptomatic. His last transthoracic echocardiogram showed no residual atrial septal or fistulous shunt, with almost normal right-sided chamber dimensions. However, his right coronary artery remains very enlarged ( $7 \mathrm{~mm}, Z$-score 12.2$)$, for which the patient remains on double antiaggregation therapy.

\section{Discussion}

Congenital coronary artery fistulas are defined as a direct communication between an epicardial coronary artery and a cardiac chamber, great vessel or other vascular structures, bypassing the myocardial capillary bed. ${ }^{1,2}$ These abnormal communications are rare in childhood, with an incidence of $1-2 \%$ in the general population. ${ }^{3}$ A coronary artery aneurysm is defined as a dilatation of coronary artery with a diameter greater than 1.5 times the diameter of the adjoining normal coronary artery. It is also rare, with a reported incidence of $\sim 1 \%$. ${ }^{4}$

The fistulae may be isolated $(55-80 \%)$, or associated with other congenital cardiac anomalies $(20-45 \%)$ or may be superimposed on coronary artery disease $(35 \%)$. The coronary artery fistulas may be single $(74-90 \%)$ or multiple $(10-16 \%)$. The origin of the fistulous communication can be the right coronary artery (50-60\%), left anterior descending coronary artery $(25-42 \%)$, or circumflex coronary artery $(18 \%)$. Its origin from the main left coronary artery is rare. ${ }^{3}$ As observed in our case, in more than $90 \%$ of the cases, the fistula terminates in the right side of the heart $(40 \%$ in the ventricle, $30 \%$ in the atrium, and $20 \%$ in the pulmonary artery). ${ }^{3}$ It rarely terminates on the left side of the heart, but when it does the majority enters the left atrium. ${ }^{5}$ 
No definite pathogenetic explanation has been described, but coronary artery fistulas may develop upon enlargement of the capillary network during embryogenesis, or when the main coronary arteries remained attached to the pulmonary trunk at the time of their segmentation. ${ }^{2}$

Patients younger than 20 years are mostly asymptomatic. However, the incidence of symptoms and complications increases over time, depending on the location, the amount of left to right shunt, and the presence of coronary steal phenomenon - due to blood shunting away from the myocardium. ${ }^{1,6}$ The symptoms and complications of untreated patients include dyspnoea, arrhythmias, congestive heart failure - when drainage occurs into the right system, resulting in pulmonary congestion and hypertension - subacute infective endocarditis, aneurysm formation, myocardial infarction, myocardial rupture, or sudden death. $^{1-3}$ The predominant physical finding is a continuous murmur at the second/third right or left parasternal border, representing the systolic-diastolic flow from the fistulae. ${ }^{1}$

A chest X-ray may show cardiomegaly and pulmonary plethora. ${ }^{3}$ The electrocardiogram is usually normal. Two-dimensional transthoracic echocardiogram can identify a dilated coronary artery, with Doppler evidence of turbulent flow at the termination site. However, it is operator dependent, and evaluation may be incomplete because of suboptimal acoustic window. ${ }^{7,8}$ The diagnostic "gold standard" remains coronary angiography, which allows haemodynamic assessment, shunt quantification, fistula characterisation, and description of the coronary artery morphology. However, it is an invasive examination; selective catheterisation of the fistulas may be difficult and the distal coronary artery may not be well evaluated because of the steal phenomenon, occurring at the proximal fistula. ${ }^{3,7}$ Cardiac computed tomography angiogram and magnetic resonance imaging are alternative diagnostic methods.

Owing to the potential adverse events and the low spontaneous closure rate $(1-2 \%)$, the current management is elective closure of the fistulas during childhood, even in asymptomatic patients. ${ }^{3,6}$ Surgical treatment is also advocated in symptomatic patients, those with large shunts $(\mathrm{Qp} / \mathrm{Qs}>1.5)$, in cases with tortuous or aneurysmatic fistulae and concomitant cardiac pathology. ${ }^{2,9}$ The most frequent surgical technique includes fistula ligation at its most proximal and distal ends, without jeopardising flow in the normal arteries. ${ }^{5}$ The surgical mortality in patients younger than 20 years is $1-2 \%$.
Significant surgical complications - arrhythmias, myocardial ischaemia, and complications related to cardiopulmonary bypass - occur in $15 \%$ of all the patients, but in only $7 \%$ of young patients. ${ }^{6}$

If the fistula is simple and single, transcatheter percutaneous closure can be an alternative option to surgery and has been increasingly used, especially among children., ${ }^{3,6}$ Complications include device migration, and currently there are no long term follow-up data regarding clot propagation and recurrence of the fistulas. ${ }^{3,6}$ In our case, device closure was not attempted as the coronary anatomy was considered unsuitable and also because there was concomitant congenital heart disease, both of which corrected during the same surgical procedure.

\section{Acknowledgements}

None.

\section{Financial Support}

This research received no specific grant from any funding agency, commercial or not-for-profit sectors.

\section{Conflicts of Interest}

None.

\section{References}

1. Kim SH, Ko JS, Yoon HJ, et al. A case of coronary arteriovenous fistula associated with giant coronary artery aneurysm. J Cardiovasc Ultrasound 2009; 17: 70-72.

2. Esmaeilzadehl M, Khaledifar A, Usefi A, et al. Right coronary artery-to-pulmonary artery fistula, the role of echocardiography. Iran Cardiovas Res J 2007; 1: 50-52.

3. Chowdhury UK, Rizhvi A, Sheil A, et al. Successful surgical correction of a patient with congenital coronary arteriovenous fistula between left main coronary artery and right superior cavo-atrial junction. Hellenic J Cardiol 2009; 50: 73-78.

4. Dogan A, Ozaydin M, Altinbas A, et al. A giant aneurysm of the circumflex coronary artery with fistulous connection to the coronary sinus: a case report. Int J Cardiovasc Imaging 2003; 19: 5-8.

5. Park MK. Miscellaneous Congenital Cardiac Conditions in Pediatric Cardiology for Practitioners, 5th edn. Mosby Elsevier, Philadelphia, 2008: 395-396.

6. Boening A, Scheewe J, Fischer G. Surgical therapy of a coronary artery fistula draining into the left atrium. Pediatric Cardiology 2001; 22: 412-414.

7. Gulati GS, Ramamurthy S, Sharma S. Utility of multislice computed tomography in the diagnosis of a right coronary artery fistula to the right atrium. J Postgrad Med 2007; 53: 191-192.

8. Zahn EM, Smallhorn JF, Egger G, et al. Echocardiographic diagnosis of fistula between the left circumflex coronary artery and the left atrium. Pediatr Cardiol 1992; 13: 178-180.

9. Mandegar MH, Saidi B, Roshanali F. Right coronary artery fistula to the right atrium. Pediatr Cardiol 2011; 32: 1053-1054. 